\title{
On the Identification of Coupled Pitch and Heave Motions Using Opposition-Based Particle Swarm Optimization
}

\author{
Yuntao Dai, ${ }^{1}$ Liqiang Liu, ${ }^{2}$ and Shanshan Feng ${ }^{1}$ \\ ${ }^{1}$ College of Science, Harbin Engineering University, 145 Nantong Street, Heilongjiang 150001, China \\ ${ }^{2}$ College of Automation, Harbin Engineering University, 145 Nantong Street, Heilongjiang 150001, China \\ Correspondence should be addressed to Yuntao Dai; peach0040@126.com
}

Received 12 December 2013; Accepted 7 February 2014; Published 9 April 2014

Academic Editor: Rongni Yang

Copyright (c) 2014 Yuntao Dai et al. This is an open access article distributed under the Creative Commons Attribution License, which permits unrestricted use, distribution, and reproduction in any medium, provided the original work is properly cited.

\begin{abstract}
A mathematical model must be established to study the motions of ships in order to control them effectively. An assessment of the model depends on the accuracy of hydrodynamic parameters. An algorithm for the parameter identification of the coupled pitch and heave motions in ships is, thus, put forward in this paper. The algorithm proposed is based on particle swarm optimization (PSO) and the opposition-based learning theory known as opposition-based particle swarm optimization (OPSO). A definition of the opposition-based learning algorithm is given first of all, with ideas on how to improve this algorithm and its process being presented next. Secondly, the design of the parameter identification algorithm is put forward, modeling the disturbing force and disturbing moment of the identification system and the output parameters of the identification system. Then, the problem involving the hydrodynamic parameters of motions is identified and the coupled pitch and heave motions of a ship described as an optimization problem with constraints. Finally, the numerical simulations of different sea conditions with unknown parameters are carried out using the PSO and OPSO algorithms. The simulation results show that the OPSO algorithm is relatively stable in terms of the hydrodynamic parameters identification of the coupled pitch and heave motions.
\end{abstract}

\section{Introduction}

The model concerned with the motions of ships describes the process of movement of the response characteristics of the control input, and this is the precondition to understanding the motions of ships. Widely used system identification techniques for hydrodynamic parameters and the hydrodynamic parameters themselves can be identified by observing the data relating to the motions of ships. This directly establishes the mathematical model of the ship's hydrodynamic parameters and motions between the state models. Classic identification algorithms, such as the maximum likelihood identification method and the prediction error used for parameter estimation are too low in terms of sensitivity and are thus inadequate as methods used in order to solve true values. Abkowitz [1] extended the Kalman Filter method to estimate the hydrodynamic parameters of a ship's motions. Clarke et al. [2] identified the nonlinear parameters of the motions of ships by using an artificial neural network. Haddara used free attenuation from the free response-signal method to identify the roll $[3,4]$ and extended it to the parameter identification of sway and yaw coupled equations [5] and pitch and heave coupled equations [6]. Haddara and $\mathrm{Xu}$ [7] additionally put forward a ship's longitudinal motions state as a Markov chain process and, in order to simplify the longitudinal motions equation, they used a neural network for the identification of a ship's longitudinal motions parameters. Mahfouz and Haddara [8] mixed the classic recognition algorithm and neural network methods, putting forward a hydrodynamic parameter identification method using RDLRNNT technology, a method that appears to be fairly sound.

Bhattacharyya and Haddara [9] used artificial neural networks (ANN) and spectral analysis methods to identify the hydrodynamic derivatives in the mathematical model involving ship and marine vehicle motions. ANN has also some defects, however, such as bad generalization performance, easily falling into a local minimum. Luo and Zou [10] applied support vector machines (SVM) to identify the hydrodynamic derivatives of Abkowitz's model from the simulated free-running model test results and then used the regressive 
Abkowitz model to predict zigzag tests. Zhang and Zou [11] proposed a novel method of artificial intelligence technology in the shape of support vector machines in order to estimate the hydrodynamic coefficients in the mathematical models of ship maneuvering motions. A comparison between the predicted hydrodynamic forces and the test results shows that the identified hydrodynamic mathematical model has a good generalization performance.

In recent years, with the rapid development of intelligent algorithms, a number of scholars have used the swarm intelligent algorithm, applying it to hydrodynamic parameters in order to identify problems related to the motions of ships. Through the state equation of underwater bodies and observation equation, Chen et al. [12] has used intelligent recognition technology for simulation recognition of an underwater navigation body and has obtained ten hydrodynamic parameters. In 2008, Chen et al. [13] proposed a new recognition algorithm based on intelligence technology. The leastsquare criterion together with the Differential Swarm Intelligent (DS) algorithm is employed to identify hydrodynamic parameters. In 2011, Dai et al. [14, 15] used an improved PSO algorithm and continuous domain ant colony optimization algorithm to identify the hydrodynamic parameters of heave and pitch with satisfactory results. In order to determine a water diving device parameter test and for the theoretical calculation of difficult problems, Gao and $\mathrm{Li}$ [16] put forward a method based on the basic artificial bee colony algorithm and the improved artificial bee colony method used to identify a potential coefficient method. Experiments show that the use of artificial colony algorithm identification device parameters is indeed feasible.

The PSO algorithm was proposed by Kennedy and Eberhart in $1995[17,18]$. In order to overcome the existing problems in the practical use of the PSO algorithm and improve the performance of the algorithm, an improved algorithm has been put forward by number of scholars [19-26]. Generally speaking, the improved PSO algorithm strategy currently includes two aspects, namely, the improvement of the strategy of the PSO algorithm and its fusion with other algorithms. The improved PSO algorithm mainly concerns the variation of the particles, the multipopulation cooperation, and the design of parameters. These methods can potentially prevent particle aggregation and conflict, and avoid premature convergence to local optima. PSO algorithms are integrated with other algorithms, improving the strategy of the PSO. Some scholars are currently advocating the opposition-based learning particle swarm optimization algorithm. Wang et al. [21] have introduced opposition-based learning into the PSO algorithm, then they proposed opposition-based learning and the Cauchy mutation PSO algorithm (OPSO), using opposition-based learning to initialize the group. Omran and Al-Sharhan [24] have used dynamic shrinkage factors to generate opposition-based solutions. Shahzad [25] have presented three kinds of opposition-based PSO algorithm: the first version of the OPSO algorithm using only oppositionbased learning in order to initialize groups and the second version (IOPSO) in addition to opposition-based learning produced the opposition-based particles as a replacement for the worst particle in the group during each iteration. In the third version of the algorithm the initialization of oppositionbased learning is removed from IOPSO. Shi and Eberhart [26] have controlled the velocity of PSO by using oppositionbased learning and proposed the speed clamping PSO algorithm (OVCPSO) based on opposition-based learning, which achieved good results.

Owing to the limitations of conventional identification methods in coupled pitch and heave motions parameter identification, in this paper, we are proposing to identify ship coupled heave and pitch motions using opposition-based PSO. In order to achieve this, we have designed a model involving wave disturbance force and torque disturbance, using the design methods for an output parameter identification system, with an opposition-based PSO algorithm for parameter identification.

\section{Pitch and Heave Motions Model}

The coupled pitch and heave motions of a ship in a realistic sea can be described by two linear second order ordinary coupled differential equations as follows [7]:

$$
\begin{aligned}
& \left(a_{33}+m_{0}\right) \ddot{z}+b_{33} \dot{z}+c_{33} z+a_{35} \ddot{\theta}+b_{35} \dot{\theta}+c_{35} \theta \\
& =F_{R}+F_{3} \\
& a_{53} \ddot{z}+b_{53} \dot{z}+c_{53} z+\left(I_{5}+a_{55}\right) \ddot{\theta}+b_{55} \dot{\theta}+c_{55} \theta \\
& \quad=F_{R} X_{R}+M_{5},
\end{aligned}
$$

where $z$ is heave; $\theta$ is pitch; $I_{5}$ is the pitching moment of inertia; $m_{0}$ is the ship's mass; $F_{R}$ is the force of the rudder; $X_{R}$ is advanced from the rudder lifting center to the ship's center of gravity; $F_{3}$ is the disturbance force of heave; $M_{5}$ is the disturbance moment of pitch, $a_{33}$ is the added mass, $a_{35}$ and $a_{53}$ are mass moment; $a_{55}$ is the moment of inertia; $b_{33}, b_{35}$ are the damping coefficients; $c_{33}, c_{35}$ are the resilience coefficient; $b_{53}, b_{55}$ are the damping moment coefficient; $c_{53}, c_{55}$ are the righting moment coefficients; and $a_{33}, a_{35}, a_{53}, a_{55}, b_{33}, b_{35}$, $b_{53}, b_{55}, c_{33}, c_{35}, c_{53}$, and $c_{55}$ are the hydrodynamic parameters.

Set $x_{1}=z, x_{3}=\theta$ as the state variables

$$
X=\left[\begin{array}{llll}
x_{1} & x_{2} & x_{3} & x_{4}
\end{array}\right]^{T}=\left[\begin{array}{llll}
z & \dot{z} & \theta & \dot{\theta}
\end{array}\right]^{T}
$$

The system state equation is then obtained as follows:

$$
\dot{X}=A X+B u+C W .
$$

Among $A=E^{-1} A^{*}, B=E^{-1} B^{*}, C=E^{-1} C^{*}$, where

$$
E=\left(\begin{array}{cccc}
1 & 0 & 0 & 0 \\
0 & a_{33}+\Delta & 0 & a_{35} \\
0 & 0 & 1 & 0 \\
0 & a_{53} & 0 & I_{5}+a_{55}
\end{array}\right)
$$




$$
\begin{gathered}
A^{*}=\left(\begin{array}{cccc}
0 & 1 & 0 & 0 \\
-c_{33} & -b_{33} & -c_{35} & -b_{35} \\
0 & 0 & 0 & 1 \\
-c_{53} & -b_{53} & -c_{55} & -b_{55}
\end{array}\right) \\
B=\left[\begin{array}{c}
0 \\
1 \\
0 \\
X_{R}
\end{array}\right], \quad u=F_{R}, \quad C=\left[\begin{array}{ll}
0 & 0 \\
1 & 0 \\
0 & 0 \\
0 & 1
\end{array}\right], \quad W=\left[\begin{array}{c}
F_{3} \\
M_{5}
\end{array}\right]
\end{gathered}
$$

para $=\left[a_{33}, a_{35}, a_{53}, a_{55}, b_{33}, b_{35}, b_{53}, b_{55}, c_{33}, c_{35}, c_{53}, c_{55}\right]$ as the parameters to be identified, $W$ is the wave disturbing force and moment.

\section{Opposition-Based Particle Swarm Optimization}

3.1. Particle Swarm Optimization Algorithm. PSO learns from the natural phenomenon of birds looking for food made by a class of population-based stochastic global optimization techniques. With respect to birds in flight, in its initial state every bird is in a random position and flies randomly in all directions, but as time goes on, these initial random state birds form a small community, through mutual learning, mutual tracking, and self-organization, fly at the same speed in the same direction, and ultimately the entire group gathers in one place, namely, the food source.

In the PSO algorithm, each individual is called a "particle," and each particle represents a potential solution. In continuous spatial coordinates, the PSO algorithm is described as follows.

Suppose that the size of the swarm is $N$ and the search space is $D$-dimensional, then the position of the $i$ th particle is presented as $X_{i}=\left(X_{i 1}, X_{i 2}, \ldots, X_{i D}\right)$, the velocity of this particle is presented as $V_{i}=\left(V_{i 1}, V_{i 2}, \ldots, V_{i D}\right)$, the $T$ fitness value of each particle in its current position is fitness $_{i}=$ fitness $\left(X_{i}\right)$, its corresponding optimal value is $P_{i}=\left(P_{i 1}, P_{i 2}, \ldots, P_{i D}\right)$ and the population current optimal experience value is recorded as $P_{g}=\left(P_{g 1}, P_{g 2}, \ldots, P_{g D}\right)$. Each particle adjusts its speed dynamically according to the comprehensive analysis of both individual and population flying experience and flies to the best position that it and other particles have experienced. Each particle updates its speed and position according to the formula equations:

$$
\begin{gathered}
V_{i}(t+1)=w V_{i}(t)+c_{1} r_{1}\left(P_{i}(t)-X_{i}(t)\right) \\
+c_{2} r_{2}\left(P_{g}(t)-X_{i}(t)\right), \\
X_{i}(t+1)=X_{i}(t)+V_{i}(t+1),
\end{gathered}
$$

where $t$ is iterative times, $d=1,2 \cdots D . r_{1}, r_{2}$ are random numbers between 0 and 1 and $C_{1}, C_{2}$ are nonnegative constants. This is called the learning factor and each iteration step is justified accordingly.

3.2. Opposition-Based Learning. Opposition-based learning was put forward by Professor Tizhoosh [19] in 2005.
He argues that intelligent algorithms are based on a random guess value concerning the initial population, with each generation coming close to the solution with the optimal solution or a close approximation of the optimal solution eventually being found. Thus, the initial guess value greatly influences the algorithm and, if the random guess value is very close to the optimal solution, the algorithm may converge quickly, but if the value is far away from the solution or is even the reverse, the algorithm will take much more time. If the current solution and its opposite are sought simultaneously, a better solution will be chosen and the efficiency of the algorithm will be greatly enhanced. According to the theory of probability, there is a $50 \%$ probability that the current solution is more remote from the optimal solution than its opposite [20].

Definition 1 (definition of the opposite number). Let $x \in R$ be a real number within a defined interval, where $x \in[a, b]$. The opposite number $x_{0}$ can be defined as

$$
x_{0}=a+b-x .
$$

Definition 2 (definition of the opposite point). In the high dimensional space, if $p=\left(x_{1}, x_{2}, \ldots, x_{D}\right)$ is a set of points in the $D$-dimensional search space where $x_{1}, x_{2}, \ldots, x_{D} \in R$, $x_{j} \in\left[a_{j}, b_{j}\right]$, then the points in the opposition set $p_{o}=$ $\left(x_{10}, x_{20}, \ldots, x_{D o}\right)$ can be defined as

$$
x_{j o}=a_{j}+b_{j}-x_{j} .
$$

Definition 3 (opposition-based optimization). For a point in the $D$-dimensional space $X=\left(x_{1}, x_{2}, \ldots, x_{D}\right)$, suppose that $f(X)$ is the function used to measure the performance of a candidate solution, according to the opposition theorem, $X_{o}=\left(x_{10}, x_{20}, \ldots, x_{D o}\right)$ will be the opposition set for $X=$ $\left(x_{1}, x_{2}, \ldots, x_{D}\right)$, If $f\left(X_{0}\right)<f(X)$, then the set of points $X$ can be replaced by $X_{0}$, or else $X$ is maintained.

3.3. Opposition-Based Particle Swarm Optimization. In PSO, each particle adjusts its search direction on the basis of the optimum location of all particles. In the initial stage, the algorithm converges quickly but slows down later on or even stops. These particles lose the ability to evolve when the speed of all the particles approaches zero and the algorithm is thought to represent convergence. Sometimes the algorithm does not converge to global extreme values, however, not even local extreme values. This is because the high aggregate and deficiency diversity of the particles takes a long time or an infinite time to skip from the focusing point.

In order to solve this problem, the opposition-based learning mechanism is introduced into the basic PSO, and a new random optimization algorithm is constructed, the opposition-based particle swarm optimization (OPSO) algorithm. In the OPSO algorithm, a variable is set and referred to as conNum. If the global best fitness is not updated during a single iteration, then the conNum $=$ conNum +1 , and when conNum reaches a constant set number setNum, it shows that there is a high concentration of particles, and the algorithm cannot find a better solution in the current position and speed. At this point, opposition-based learning is brought 
$\% \% \quad$ Initialization

(1) Initialize swarm size $N$ and constant number $C_{1}$ and $C_{2}$; space dimension $D$; maximum

(2) Initialize the iteration number for opposition calculation conNum $=0$, setnum $=100$;

(3) for $i=1$ : particleNum

(4) initialize $X_{i}$ randomly with the search range $\left(X_{\min }, X_{\max }\right)$

(5) initialize $V_{i}$ randomly with the velocity range $\left(V_{\min }, V_{\max }\right)$

(6) End for

(7) Evaluate each particle's fitness $f_{i}=$ fitness $\left(X_{i}\right)$, and the best fitness PbestValue ${ }_{i}=f_{i}$

(8) Identify the best particle's position Pg and its fitness GbestValue $=\min \left(\right.$ PbestValue $\left._{i}\right)$; \%\%Loop

(9) While $\left(t<n_{\max }\right)$

(10) If conNum > setNum then

(11) for $i=1$ to $N$

(12) Calculate the opposite particle $X_{0}$ using (8);

(13) Evaluate fitness in opposite vector $f_{\text {io }}=\operatorname{fitness}\left(X_{i o}\right)$;

(14) end for

(15) Select $N$ fittest particles Newfitness from $F\left(f_{1}, f_{1}, \ldots, f_{N}\right)$ and $F_{0}\left(f_{1 o}, f_{1 o}, \ldots, f_{N o}\right)$ to

(16) create a population of size $N$;

(17) Else

(18) for $i=1$ to $N$

(19) Calculate particle velocity $V_{i}(t+1)$ using (5)

(20) update particle position $X_{i}(t+1)$ using (6)

(21) Evaluate newfitness ${ }_{i}=$ fitness $_{(}\left(X_{i}(t+1)\right)$

(22) End for

(23) End if

(24) update Pbest

(25) for $i=1$ to $N$

(26) if (newFitness < GbestValue)

(27) update Gbest

(28) $\quad$ conNum $=0$;

(29) end if

(30) end for

(31) $\quad$ conNum $=$ conNum +1 ;

(32) end while

Algorithm 1: The process of OPSO.

into play. According to (8) the position-based position $X_{o}$ of the particle's current position is produced, the smaller values $N$ are selected from $X$ and $X_{o}$ which have $2 N$ locations and a new swarm is then formed. If the conNum is less than setNum, then according to (5) and (6) the particle velocity and position are updated. The process of OPSO is shown in Algorithm 1.

\section{Design of Pitch and Heave Parameter Identification}

The value of pitch and heave can be measured, so select state $x_{1}$ and $x_{3}$ in (2) as the measurement state, with the observation equation as

$$
Y=H X+V, \quad H=\left(\begin{array}{llll}
1 & 0 & 0 & 0 \\
0 & 0 & 1 & 0
\end{array}\right),
$$

where $Y$ is an observation vector and $V$ is the twodimensional measurement of noise, and this is generally considered to be white noise.

Before computer simulation, it was necessary to disperse the state equation and observation equation. This paper does not consider the steering input but only the wave disturbance on the ship, with the state equation being formulated as follows:

$$
\begin{gathered}
X_{k+1}=\Phi X_{k}+\Gamma W_{k}, \\
Y_{k+1}=H X_{k+1}+V_{k+1},
\end{gathered}
$$

where, $\boldsymbol{\Phi}=e^{A T_{s}}, \Gamma=\int_{0}^{T_{s}} e^{A t} d t C$.

In this paper, the single-parameter ITTC spectrum is chosen for wave disturbance simulation, with the expression being formulated as follows:

$$
S_{\zeta}(\omega)=\frac{8.1 \times 10^{-3} g^{2}}{\omega^{5}} \exp \left(\frac{-3.11}{h_{1 / 3}^{2} \omega^{4}}\right)
$$

where $g$ is the acceleration of gravity, $w$ is the natural angular frequency, and $h_{1 / 3}$ is a third significant wave height, for a level 4 and 5 sea condition, and the ITTC recommended value is $2.5 \mathrm{~m}$ and $3.75 \mathrm{~m}$, respectively. 
By (10), the observed quantity is the function of $X$, para, namely:

$$
y_{i}=g\left(x_{i}, \operatorname{para}_{i}, W_{i}\right)+V_{i}, \quad i=1,2, \ldots, \text { dataNum, }
$$

where dataNum is the times of observation, $g(\cdot)$ is the model output vector, and $y_{i}$ is the observation vector. The minimum mean square errors between the observed data and identical value are setting as follows:

$$
\begin{array}{r}
F_{j}=\operatorname{sqrt}\left(\frac{1}{\text { dataNum }} \sum_{i=1}^{\text {dataNum }}\left(y_{i}-g\left(x_{i}, \operatorname{para}_{i}, W_{i}\right)\right)^{2}\right), \\
j=1,2 .
\end{array}
$$

The hydrodynamic parameters of our requirements should mean that the two components in (13) obtain their minimum value simultaneously under constrained conditions (10). We must set different weight coefficients to ensure that each parameter has the same effect on the objective function, taking into consideration the different order of magnitude of pitch and heave. The fitness function is shown as follows:

$$
\text { Fit }=F_{1}+\alpha F_{2},
$$

where $\alpha$ is the weight coefficient.

\section{Experiment Testing and Comparisons}

Here the experimental ship's parameters are as follows: the hull quality $m$ is $442000 \mathrm{~kg}$, the beam $B$ is $7.2 \mathrm{~m}$, and the draft is $2.25 \mathrm{~m}$. The waterline $L_{p p}$ is $60 \mathrm{~m} . I_{5}=(0.25 *$ $\left.L_{P P}\right), m_{0}=99450000\left(\mathrm{~kg} \cdot \mathrm{m}^{2}\right), X_{R}=25.8 \mathrm{~m}$, and $Z_{R}=$ $1.32 \mathrm{~m}$. The number of rudder $n$ is 2 and the hydrostatic resilience factor $C_{44}$ is 3370000 . For the ship, $C_{33}, C_{35}, C_{55}$, and $C_{53}$ are constants and can be obtained by calculating the structural parameters of the ship. $V$ is Gaussian white noise $V$ is Gaussian white noise and its covariance matrix can be taken as so that the accuracy of the sensor can be taken as $Q_{V V}=$ $\operatorname{diag}[20.3 e-42.25 e-6]$.

A comparative algorithm is the OPSO algorithm proposed in this paper and the PSO-w (PSO with inertia weight [26]), with the parameters being set as shown in Table 1 .

The identification results by PSO and OPSO are shown in Tables 2, 3, and 4, for level 4 sea condition, speed $18 \mathrm{kn}$ and course angle $90^{\circ}, 135^{\circ}$, and $180^{\circ}$, respectively.

As we can see from Table 2 to Table 4, the parameters of pitch and heave are correctly identified by using the PSO algorithm, with the OPSO algorithm clearly obtaining better results than the PSO, especially for level 4 sea condition, speed $18 \mathrm{kn}$, and course angle $135^{\circ}$, and the relative error of the OPSO is smaller than for the PSO algorithm.

From the identification of the parameters of pitch and heave motions carried out by using the OPSO algorithm, we can obtain the mathematical model for pitch and heave which is constructed according to the identification of the hydrodynamic parameters. The model in which the pitch and
TABLE 1: Parameters and their range of values used in our proposed algorithm.

\begin{tabular}{lc}
\hline Parameters & Value/range \\
\hline Population size of swarm & 100 \\
Acceleration constants $c_{1}, c_{2}$ & $c_{1}=c_{2}=1.4962$ \\
Inertia weight $w$ & {$[0.4,0.9]$} \\
Random number $r_{1}, r_{2}$ & {$[0,1]$} \\
Maximum iteration times $n_{\max }$ & 1000 \\
Range of velocity $V_{\max }$ & $\left(x_{\mathrm{Max}}-x_{\text {Min }}\right) / 2$ \\
setNum for OPSO & 100 \\
Sampling dot number dataNum & 200 \\
\hline
\end{tabular}

heave for sea condition 4 , speed $18 \mathrm{kn}$, and course angle $90^{\circ}$, $135^{\circ}$, and $180^{\circ}$ is shown in Figures 1, 2, and 3, respectively. There are three curves in each diagram; the first curve "- $\square$ - " represents the observed values of the coupled pitch and heave motions, the second curve " $-\mathbf{\nabla}-$ " represents the model constructed by PSO-w, and the last curve "-_represents the model output values constructed by OPSO.

It is obvious in these figures that there was an agreement between the pitch and heave model estimated by the PSO$\mathrm{w}$ model and observed values for a level 4 sea condition, ship speed $18 \mathrm{kn}$, and course angle $90^{\circ}$ and $180^{\circ}$, but when the course angle was $90^{\circ}$, the results were not accurate. However, the model estimated by the OPSO algorithm tallies completely with the observed values.

Tables 5, 6, and 7 and Figures 4, 5, and 6 show similar results for a level 5 sea condition. It is clear in Tables 5-7 that the parameters identified by the OPSO algorithm are close to the real ones while those identified by PSO-w are not, especially the results for level 5 sea condition, ship speed $18 \mathrm{kn}$, and course angle 90 degrees, as the parameters have greater relative errors. Because of this, the corresponding pitch and heave models do not tally with the observed values. However, for other course angles, both the PSO-w and OPSO algorithms agree with observed values.

\section{Conclusions}

The identification of the hydrodynamic parameters of ships is an important way of obtaining these parameters. In this paper, we have used OPSO to design the method of identifying the hydrodynamic parameters of the coupled pitch and heave motions of ships. This paper introduces in detail the opposition-basedlearning algorithm and puts forward an improved idea and process for the oppositionbased algorithm. In addition, this paper introduces the process involving the hydrodynamic parameter identification algorithm. Here, we have established wave disturbance as the model input, with the algorithm's fitness function being the output model. The hydrodynamic parameter identification problem was then converted into a constrained optimization problem and the OPSO algorithm was used to find the optimal solution. Finally, we made use of computer simulation, with the simulation results showing that the OPSO algorithm is relatively stable in terms of identifying the hydrodynamic 
TABLE 2: Parameter identification of pitch and heave motions for level 4 sea condition, ship speed $18 \mathrm{kn}$ and course angle $90^{\circ}$.

\begin{tabular}{|c|c|c|c|c|c|}
\hline \multirow{2}{*}{ Parameters } & \multirow{2}{*}{ Theoretical values } & \multicolumn{2}{|c|}{ PSO-w } & \multicolumn{2}{|c|}{ OPSO } \\
\hline & & Identification value & Relative error & Identification value & Relative error \\
\hline$a_{33}$ & $8.045 E+05$ & $9.446 E+05$ & $17.42 \%$ & $8.141 E+05$ & $1.19 \%$ \\
\hline$b_{33}$ & $9.369 E+05$ & $7.901 E+05$ & $15.66 \%$ & $9.447 E+05$ & $0.83 \%$ \\
\hline$a_{35}$ & $7.753 E+06$ & $8.902 E+06$ & $14.82 \%$ & $8.037 E+06$ & $3.66 \%$ \\
\hline$b_{35}$ & $1.159 E+07$ & $1.480 E+07$ & $27.72 \%$ & $1.222 E+07$ & $5.39 \%$ \\
\hline$a_{55}$ & $2.595 E+08$ & $2.830 E+08$ & $9.05 \%$ & $2.706 E+08$ & $4.27 \%$ \\
\hline$b_{55}$ & $2.885 E+08$ & $4.077 E+08$ & $41.31 \%$ & $2.892 E+08$ & $0.26 \%$ \\
\hline$a_{53}$ & $1.479 E+07$ & $1.777 E+07$ & $20.17 \%$ & $1.500 E+07$ & $1.39 \%$ \\
\hline$b_{53}$ & $3.324 E+06$ & $2.296 E+06$ & $30.91 \%$ & $3.068 E+06$ & $7.70 \%$ \\
\hline
\end{tabular}

TABle 3: Parameter identification of pitch and heave motions for level 4 sea condition, ship speed $18 \mathrm{kn}$ and course angle $135^{\circ}$.

\begin{tabular}{|c|c|c|c|c|c|}
\hline \multirow{2}{*}{ Parameters } & \multirow{2}{*}{ Theoretical values } & \multicolumn{2}{|c|}{ PSO-w } & \multicolumn{2}{|c|}{ OPSO } \\
\hline & & Identification value & Relative error & Identification value & Relative error \\
\hline$a_{33}$ & $6.311 E+05$ & $5.569 E+05$ & $11.77 \%$ & $6.177 E+05$ & $2.12 \%$ \\
\hline$b_{33}$ & $8.033 E+05$ & $7.635 E+05$ & $4.96 \%$ & $8.245 E+05$ & $2.64 \%$ \\
\hline$a_{35}$ & $3.281 E+06$ & $5.248 E+06$ & $59.95 \%$ & $3.057 E+06$ & $6.84 \%$ \\
\hline$b_{35}$ & $9.429 E+06$ & $7.879 E+06$ & $16.44 \%$ & $9.598 E+06$ & $1.80 \%$ \\
\hline$a_{55}$ & $1.525 E+08$ & $1.541 E+08$ & $1.01 \%$ & $1.533 E+08$ & $0.52 \%$ \\
\hline$b_{55}$ & $2.091 E+08$ & $2.716 E+08$ & $29.88 \%$ & $2.115 E+08$ & $1.14 \%$ \\
\hline$a_{53}$ & $7.865 E+06$ & $9.509 E+06$ & $20.90 \%$ & $7.772 E+06$ & $1.18 \%$ \\
\hline$b_{53}$ & $2.267 E+06$ & $1.616 E+06$ & $28.72 \%$ & $2.423 E+06$ & $6.87 \%$ \\
\hline
\end{tabular}

TABle 4: Parameter identification of pitch and heave motions for level 4 sea condition, ship speed $18 \mathrm{kn}$ and course angle $180^{\circ}$.

\begin{tabular}{|c|c|c|c|c|c|}
\hline \multirow{2}{*}{ Parameters } & \multirow{2}{*}{ Theoretical values } & \multicolumn{2}{|c|}{ PSO-w } & \multicolumn{2}{|c|}{ OPSO } \\
\hline & & Identification value & Relative error & Identification value & Relative error \\
\hline$a_{33}$ & $6.101 E+05$ & $5.815 E+05$ & $4.69 \%$ & $6.148 E+05$ & $0.77 \%$ \\
\hline$b_{33}$ & $7.400 E+05$ & $7.782 E+05$ & $-5.16 \%$ & $7.366 E+05$ & $0.46 \%$ \\
\hline$a_{35}$ & $2.663 E+06$ & $2.396 E+06$ & $10.03 \%$ & $2.758 E+06$ & $3.56 \%$ \\
\hline$b_{35}$ & $8.951 E+06$ & $8.835 E+06$ & $1.30 \%$ & $8.873 E+06$ & $0.87 \%$ \\
\hline$a_{55}$ & $1.377 E+08$ & $1.348 E+08$ & $2.14 \%$ & $1.367 E+08$ & $0.74 \%$ \\
\hline$b_{55}$ & $1.873 E+08$ & $1.944 E+08$ & $-3.79 \%$ & $1.838 E+08$ & $1.87 \%$ \\
\hline$a_{53}$ & $6.611 E+06$ & $6.594 E+06$ & $0.25 \%$ & $6.568 E+06$ & $0.65 \%$ \\
\hline$b_{53}$ & $2.356 E+06$ & $3.123 E+06$ & $-32.57 \%$ & $2.113 E+06$ & $10.32 \%$ \\
\hline
\end{tabular}

TABle 5: Parameter identification for pitch and heave motions for level 5 sea condition, ship speed $18 \mathrm{kn}$ and course angle $90^{\circ}$.

\begin{tabular}{|c|c|c|c|c|c|}
\hline \multirow{2}{*}{ Parameters } & \multirow{2}{*}{ Theoretical values } & \multicolumn{2}{|c|}{ PSO-w } & \multicolumn{2}{|c|}{ OPSO } \\
\hline & & Identification value & Relative error & Identification value & Relative error \\
\hline$a_{33}$ & $9.375 E+05$ & $1.178 E+06$ & $25.64 \%$ & $9.111 E+05$ & $2.82 \%$ \\
\hline$b_{33}$ & $9.089 E+05$ & $8.644 E+05$ & $4.89 \%$ & $9.635 E+05$ & $6.01 \%$ \\
\hline$a_{35}$ & $1.137 E+07$ & $2.102 E+07$ & $-84.86 \%$ & $1.030 E+07$ & $9.39 \%$ \\
\hline$b_{35}$ & $1.274 E+07$ & $1.831 E+07$ & $43.71 \%$ & $1.295 E+07$ & $1.65 \%$ \\
\hline$a_{55}$ & $3.613 E+08$ & $4.017 E+08$ & $11.19 \%$ & $3.532 E+08$ & $2.25 \%$ \\
\hline$b_{55}$ & $3.202 E+08$ & $4.593 E+08$ & $43.43 \%$ & $3.428 E+08$ & $7.07 \%$ \\
\hline$a_{53}$ & $1.942 E+07$ & $2.430 E+07$ & $25.14 \%$ & $1.964 E+07$ & $1.11 \%$ \\
\hline$b_{53}$ & $4.641 E+06$ & $3.829 E+06$ & $17.50 \%$ & $5.348 E+06$ & $-15.24 \%$ \\
\hline
\end{tabular}




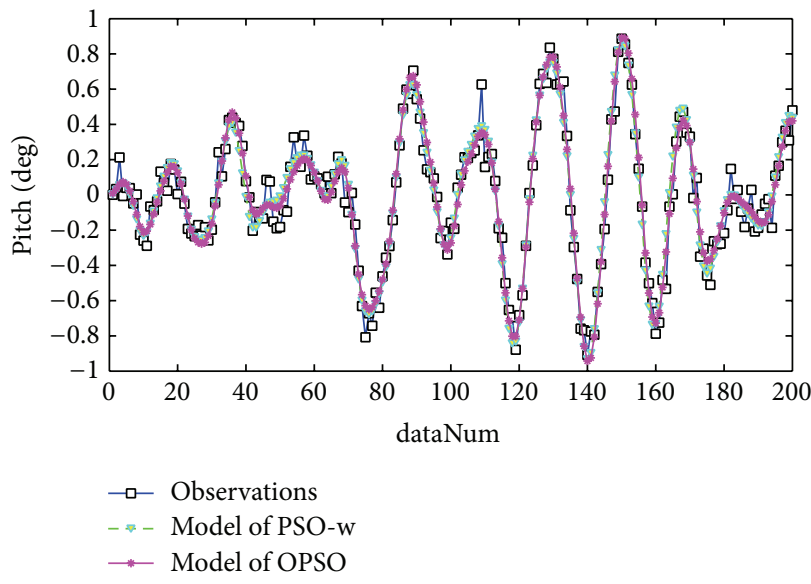

(a) Pitch

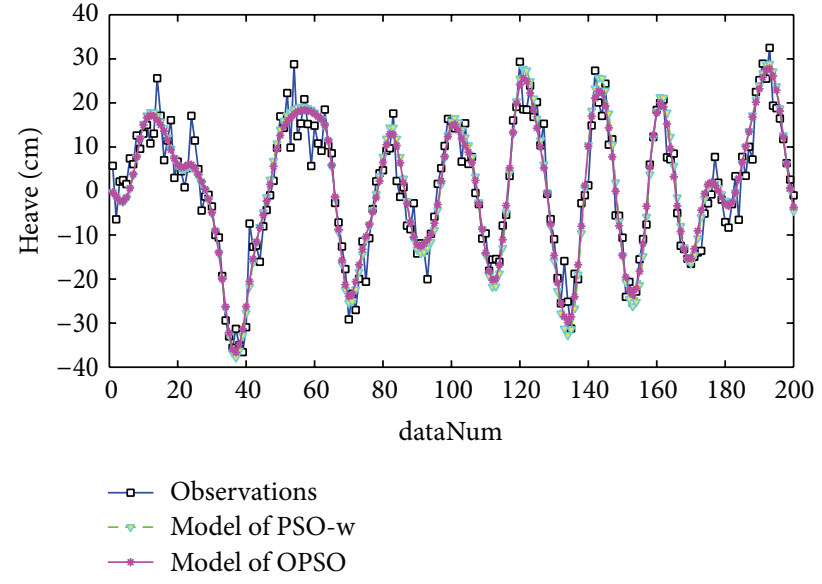

(b) Heave

FIGURE 1: Pitch and heave observed value. Model identified by PSO-w and model identified by OPSO for level 4 sea condition, ship speed $18 \mathrm{kn}$ and course angle $90^{\circ}$.

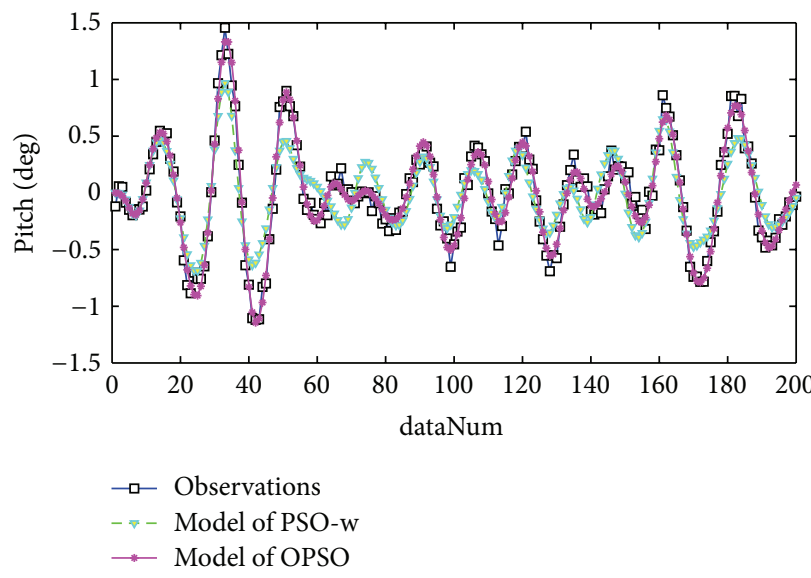

(a) Pitch

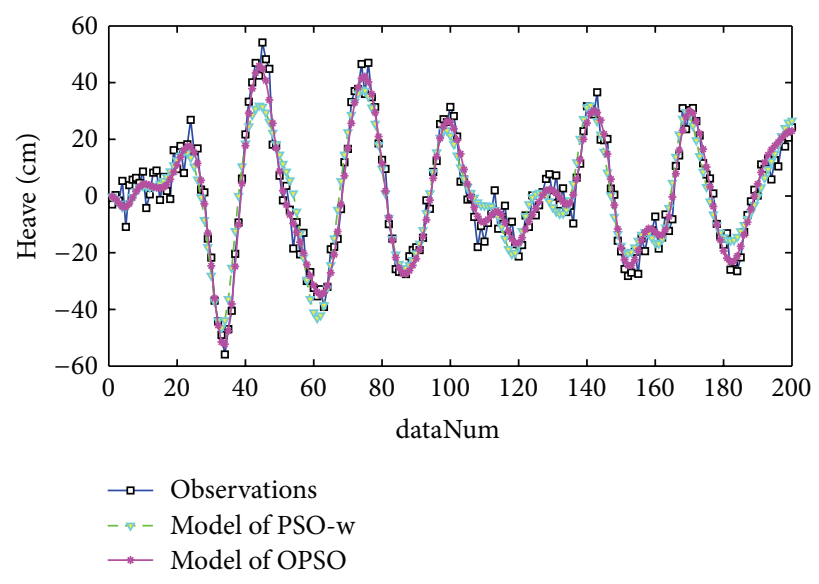

(b) Heave

FIGURE 2: Pitch and heave observed value. Model identified by PSO-w and model identified by OPSO for level 4 sea condition, ship speed $18 \mathrm{kn}$ and course angle $135^{\circ}$.

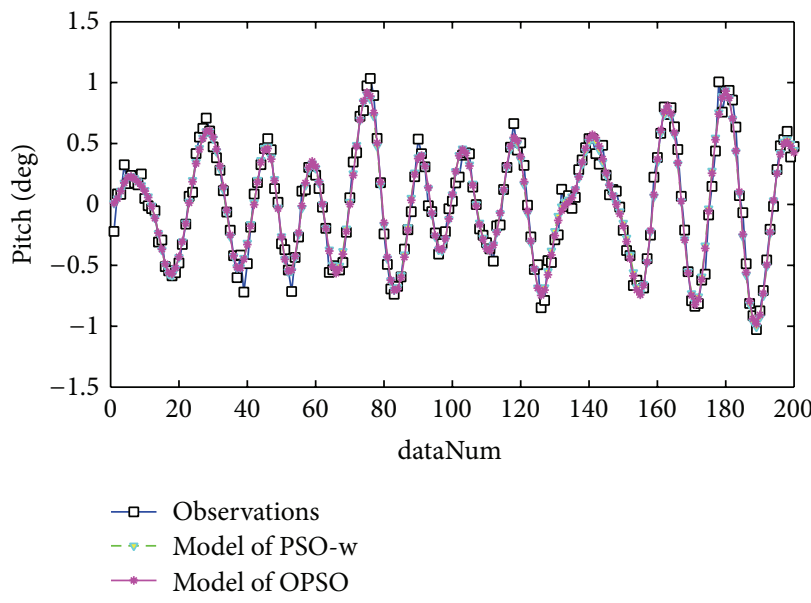

(a) Pitch

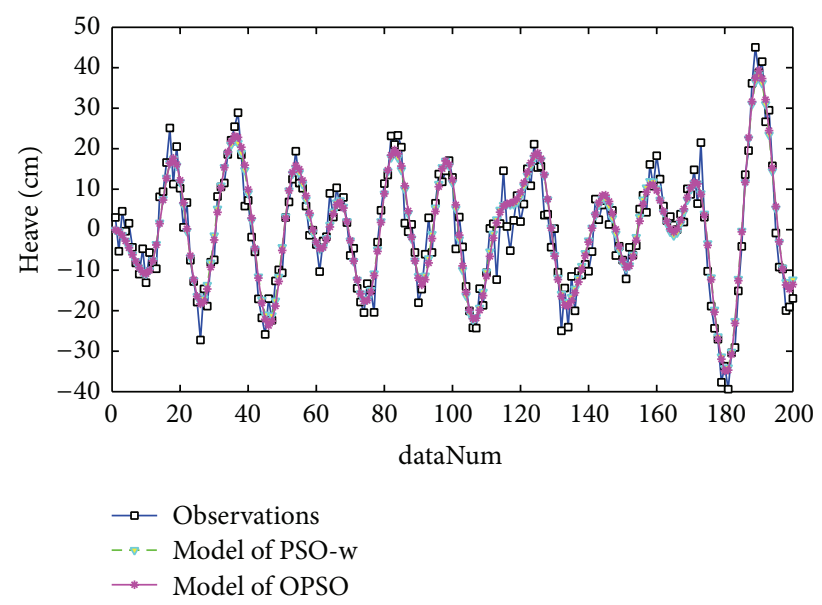

(b) Heave

FIGURE 3: Pitch and heave observed value. Model identified by PSO-w and model identified by OPSO for level 4 sea condition, ship speed $18 \mathrm{kn}$ and course angle $180^{\circ}$. 


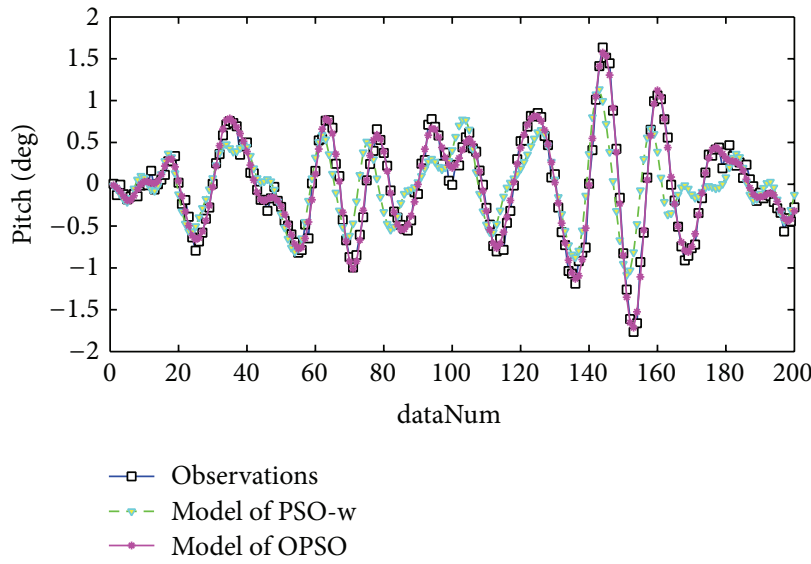

(a) Pitch

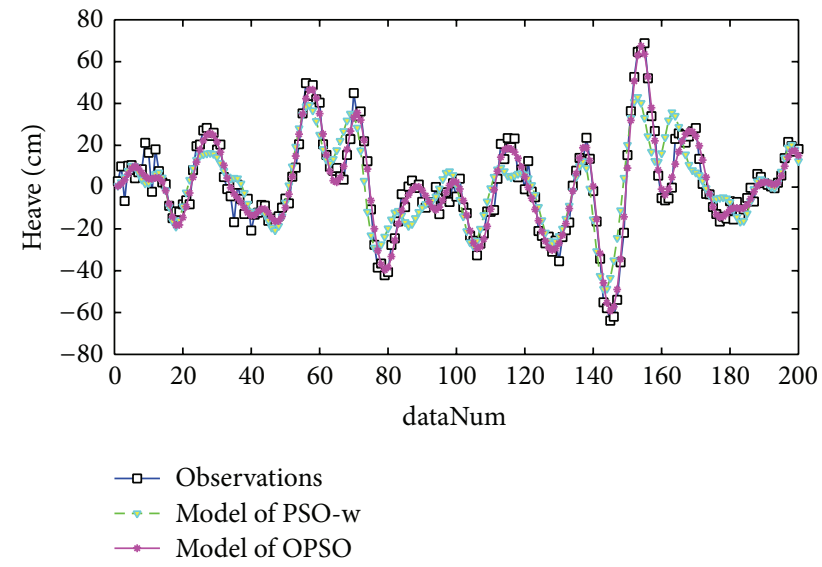

(b) Heave

FIGURE 4: Pitch and heave observed value. Model identified by PSO-w and model identified by OPSO for level 5 sea condition, ship speed $18 \mathrm{kn}$ and course angle $90^{\circ}$.

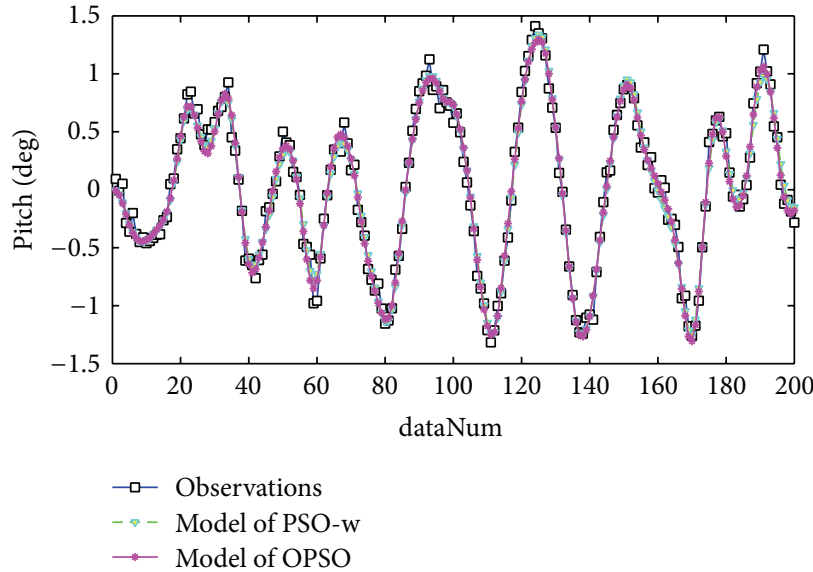

(a) Pitch

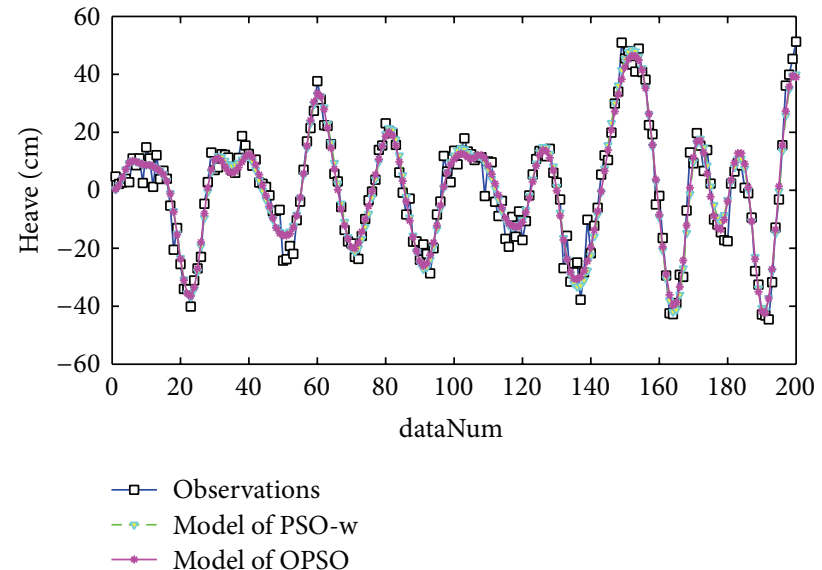

(b) Heave

Figure 5: Pitch and heave observed value. Model identified by PSO-w and model identified by OPSO for level 5 sea condition, ship speed $18 \mathrm{kn}$ and course angle $135^{\circ}$.

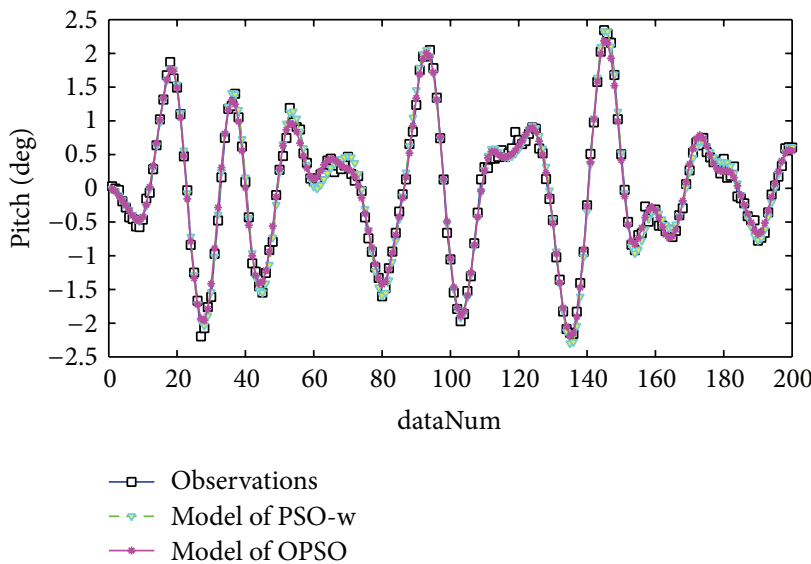

(a) Pitch

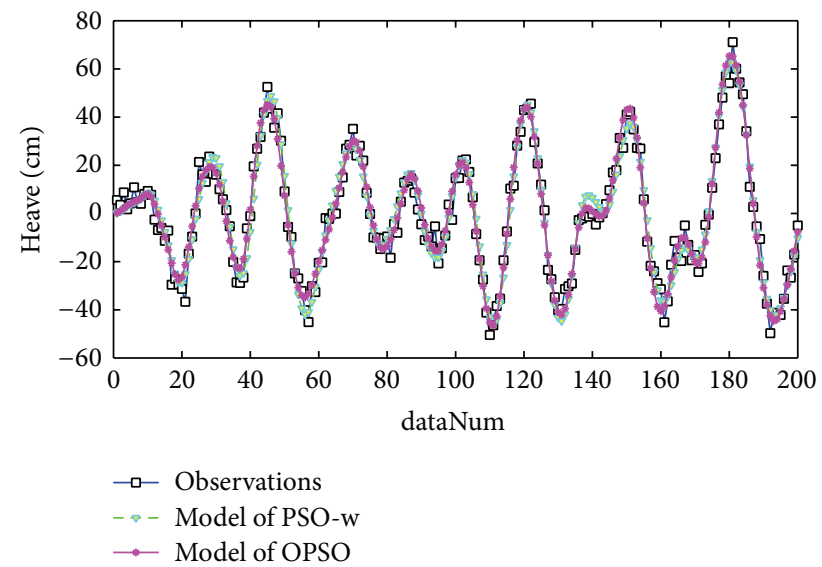

(b) Heave

FIGURE 6: Pitch and heave observed value. Model identified by PSO-w and model identified by OPSO for level 5 sea condition, ship speed $18 \mathrm{kn}$ and course angle $180^{\circ}$. 
TABLE 6: Parameter identification of pitch and heave motions for level 5 sea condition, ship speed $18 \mathrm{kn}$ and course angle $135^{\circ}$.

\begin{tabular}{|c|c|c|c|c|c|}
\hline \multirow{2}{*}{ Parameters } & \multirow{2}{*}{ Theoretical values } & \multicolumn{2}{|c|}{ PSO-w } & \multicolumn{2}{|c|}{ OPSO } \\
\hline & & Identification value & Relative error & Identification value & Relative error \\
\hline$a_{33}$ & $7.202 E+05$ & $7.515 E+05$ & $-4.34 \%$ & $7.106 E+05$ & $1.34 \%$ \\
\hline$b_{33}$ & $8.553 E+05$ & $8.283 E+05$ & $3.15 \%$ & $8.921 E+05$ & $4.31 \%$ \\
\hline$a_{35}$ & $5.539 E+06$ & $5.923 E+06$ & $-6.93 \%$ & $5.426 E+06$ & $2.05 \%$ \\
\hline$b_{35}$ & $1.046 E+07$ & $9.616 E+06$ & $8.10 \%$ & $1.043 E+07$ & $0.35 \%$ \\
\hline$a_{55}$ & $2.051 E+08$ & $2.086 E+08$ & $-1.70 \%$ & $2.079 E+08$ & $1.34 \%$ \\
\hline$b_{55}$ & $2.451 E+08$ & $2.473 E+08$ & $-0.90 \%$ & $2.467 E+08$ & $0.64 \%$ \\
\hline$a_{53}$ & $1.124 E+07$ & $1.115 E+07$ & $0.82 \%$ & $1.167 E+07$ & $3.85 \%$ \\
\hline$b_{53}$ & $2.876 E+06$ & $2.297 E+06$ & $20.13 \%$ & $2.895 E+06$ & $0.64 \%$ \\
\hline
\end{tabular}

TABLE 7: Parameter identification of pitch and heave motions for level 5 sea condition, ship speed $18 \mathrm{kn}$ and course angle $180^{\circ}$.

\begin{tabular}{|c|c|c|c|c|c|}
\hline \multirow{2}{*}{ Parameters } & \multirow{2}{*}{ Theoretical values } & \multicolumn{2}{|c|}{ PSO-w } & \multicolumn{2}{|c|}{ OPSO } \\
\hline & & Identification value & Relative error & Identification value & Relative error \\
\hline$a_{33}$ & $6.787 E+05$ & $6.812 E+05$ & $-0.38 \%$ & $6.762 E+05$ & $0.37 \%$ \\
\hline$b_{33}$ & $8.182 E+05$ & $7.952 E+05$ & $2.82 \%$ & $7.939 E+05$ & $2.97 \%$ \\
\hline$a_{35}$ & $4.428 E+06$ & $3.406 E+06$ & $23.09 \%$ & $4.638 E+06$ & $4.74 \%$ \\
\hline$b_{35}$ & $9.914 E+06$ & $9.902 E+06$ & $0.12 \%$ & $9.849 E+06$ & $0.66 \%$ \\
\hline$a_{55}$ & $1.783 E+08$ & $1.798 E+08$ & $-0.89 \%$ & $1.742 E+08$ & $2.30 \%$ \\
\hline$b_{55}$ & $2.246 E+08$ & $2.317 E+08$ & $-3.17 \%$ & $2.284 E+08$ & $1.70 \%$ \\
\hline$a_{53}$ & $9.511 E+06$ & $9.738 E+06$ & $-2.38 \%$ & $9.351 E+06$ & $1.69 \%$ \\
\hline$b_{53}$ & $2.664 E+06$ & $2.228 E+06$ & $16.37 \%$ & $2.974 E+06$ & $11.62 \%$ \\
\hline
\end{tabular}

parameters connected with the problem of the coupled pitch and heave motions of ships. In addition, the identified coupled pitch and heave model values and the observed values are consistent. This method may provide a new solution for the identification of coupled pitch and heave motions. This paper has not taken the disturbance of the rudder angle into consideration, something which the author intends to research in due course.

\section{Conflict of Interests}

The authors declare that there is no conflict of interests regarding the publication of this paper.

\section{Acknowledgments}

This work has been financially supported by the National Natural Science Foundation of China under the Grant 51109041 and the fundamental research funds for the central universities.

\section{References}

[1] M. A. Abkowitz, "Measurement of hydrodynamic characteristics from ship manoeuvring trials by system identification," Transactions of the Society of Naval Architects and Marine Engineers, vol. 88, pp. 283-318, 1980.
[2] D. P. Clarke, P. Gedling, and G. Hine, "The application of manoeuvring criteria in hull design using linear theory," Transactions of the Royal Institution of Naval Architects, vol. 125, pp. $45-68,1982$.

[3] M. R. Haddara, "On the use of neural network techniques for the identification of ship stability parameters at sea," in Proceedings of the 14th International Conference on Offshore Mechanics and Arctic Engineering, vol. 2, pp. 127-135, 1995.

[4] M. R. Haddara, "On the random decrement for nonlinear rolling motion," in Proceedings of the 12th International Conference on Offshore Mechanics and Arctic Engineering, vol. 2, pp. 283-288, 1992.

[5] M. R. Haddara and Y. Wang, "Parametric identification of coupled sway and yaw motions," in Proceedings of the 15th International Conferece on Offshore Mechanics and Arctic Engineering, vol. 1, pp. 267-273, 1996.

[6] M. R. Haddara and J. S. Xu, "On the use of random decrement in the identification of two degrees of freedom systems," in Proceedings of the Canadian Society for Mechanical Engineering Forum (CSME '98), vol. 4, pp. 499-507, Ryerson Polytechnic University, 1998.

[7] M. R. Haddara and J. Xu, "On the identification of ship coupled heave-pitch motions using neural networks," Ocean Engineering, vol. 26, no. 5, pp. 381-400, 1998.

[8] A. B. Mahfouz and M. R. Haddara, "Effect of the damping and excitation on the identification of the hydrodynamic parameters for an underwater robotic vehicle," Ocean Engineering, vol. 30, no. 8, pp. 1005-1025, 2003. 
[9] S. K. Bhattacharyya and M. R. Haddara, "Parametric identification for nonlinear ship maneuvering," Journal of Ship Research, vol. 50, no. 3, pp. 197-207, 2006.

[10] W. L. Luo and Z. J. Zou, "Parametric identification of ship maneuvering models by using support vector machines," Journal of Ship Research, vol. 53, no. 1, pp. 19-30, 2009.

[11] X. G. Zhang and Z. J. Zou, "Estimation of the hydrodynamic coefficients from captive model test results by using support vector machines," Ocean Engineering, vol. 73, pp. 25-31, 2013.

[12] W. Q. Chen, K. Yan, G. J. Shi, S. T. Wang, and Z. Y. Liu, "Research of hydrodynamic parameter identification for underwater vehicle using swarm intelligence algorithm," Journal of Ship Mechanics, vol. 11, no. 1, pp. 40-46, 2007.

[13] W. Q. Chen, K. Yan, and B. S. Wang, "Intelligent identification of hydrodynamic parameters of navigating body," Journal of Ship Mechanics, vol. 12, no. 2, pp. 204-210, 2008.

[14] Y. T. Dai, X. R. Zhao, and L. Q. Liu, "Parameter identification of ship longitudinal motions using continuous domains ant colony algorithm," Computer Engineering, vol. 26, no. 16, pp. 23-24, 2010.

[15] Y. T. Dai, X. R. Zhao, and L. Q. Liu, "Parameter identification of ship vertical motions using improved particle swarm optimization," Journal of Ship Mechanics, vol. 14, no. 1-2, pp. 44-50, 2010.

[16] M. S. Gao and S. M. Li, "Identification on hydrodynamic coefficients of underwater vehicle with the ABC algorithm," Journal of Harbin Engineering University, vol. 34, no. 8, pp. 1-5, 2013.

[17] J. Kennedy and R. C. Eberhart, "Particle swarm optimization," in Proceedings of the IEEE International Conference on Neural Networks, pp. 1942-1948, 1995.

[18] R. Eberhart and J. Kennedy, "A new optimizer using particle swarm theory," in Proceedings of the 6th International Symposium on Micro Machine and Human Science, pp. 39-43, 1995.

[19] H. R. Tizhoosh, "Opposition-based learning: a new scheme for machine intelligence," in Proceedings of the International Conference on Computational Intelligence for Modelling, Control and Automation (CIMCA '05), vol. 1, pp. 695-701, Vienna, Austria, 2005.

[20] S. Rahnamayan, H.R. Tizhoosh, and M. M. Salama, "Opposition-based differential evolution," IEEE Transactions on Evolutionary Computation, vol. 12, no. 1, pp. 64-79, 2008.

[21] H. Wang, H. Li, Y. Liu, C. Li, and S. Zeng, "Opposition-based particle swarm algorithm with cauchy mutation," in Proceedings of the IEEE Congress on Evolutionary Computation (CEC '07), pp. 4750-4756, Singapore, 2007.

[22] J. Kennedy, "Particle swarm: social adaptation of knowledge," in Proceedings of the IEEE International Conference on Evolutionary Computation, pp. 303-308, 1997.

[23] H. Lin and H. Xingshi, "A novel opposition-based particle swarm optimization for noisy problems," in Proceedings of the 3rd International Conference on Natural Computation (ICNC '07), pp. 624-629, Haikou, China, 2007.

[24] M. G. H. Omran and S. Al-Sharhan, "Using opposition-based learning to improve the performance of particle swarm optimization," in Proceedings of the IEEE Swarm Intelligence Symposium (SIS '08), pp. 1-6, Saint Louis, Mo, USA, 2008.

[25] F. Shahzad, A. R. Baig, S. Masood, M. Karman, and N. Naveed, "Opposition-based particle swarm optimization with velocity clamping (OVCPSO)," in Advances in Computational Intelligence, vol. 116 of Advances in Intelligent and Soft Computing, pp. 339-348, 2009.
[26] Y. Shi and R. C. Eberhart, "A modified particle swarm optimizer," in Proceedings of the IEEE International Conference on Evolutionary Computation, IEEE World Congress on Computational Intelligence, pp. 69-73, Anchorage, Alaska, USA, 1998. 


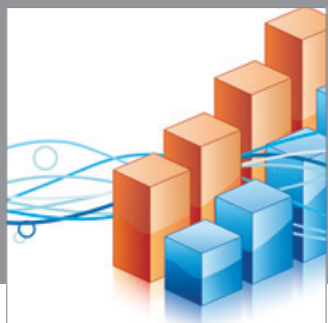

Advances in

Operations Research

mansans

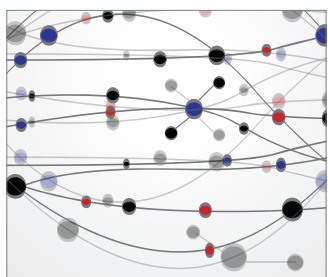

The Scientific World Journal
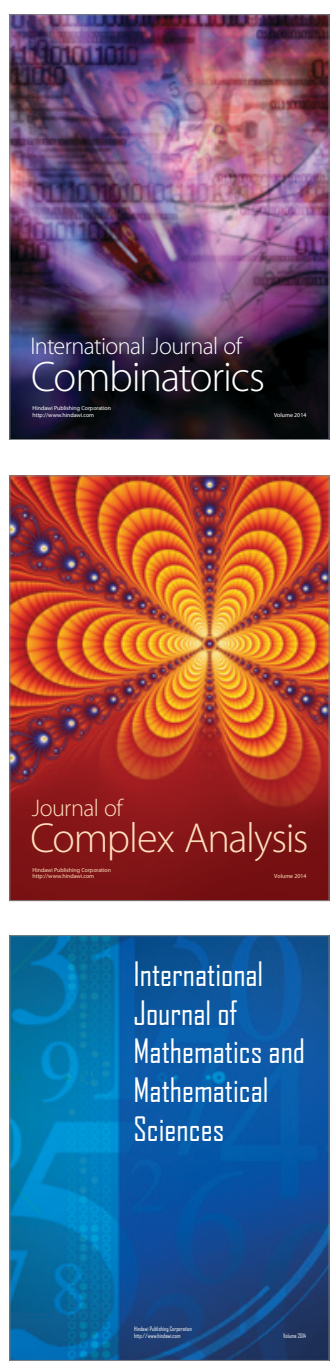
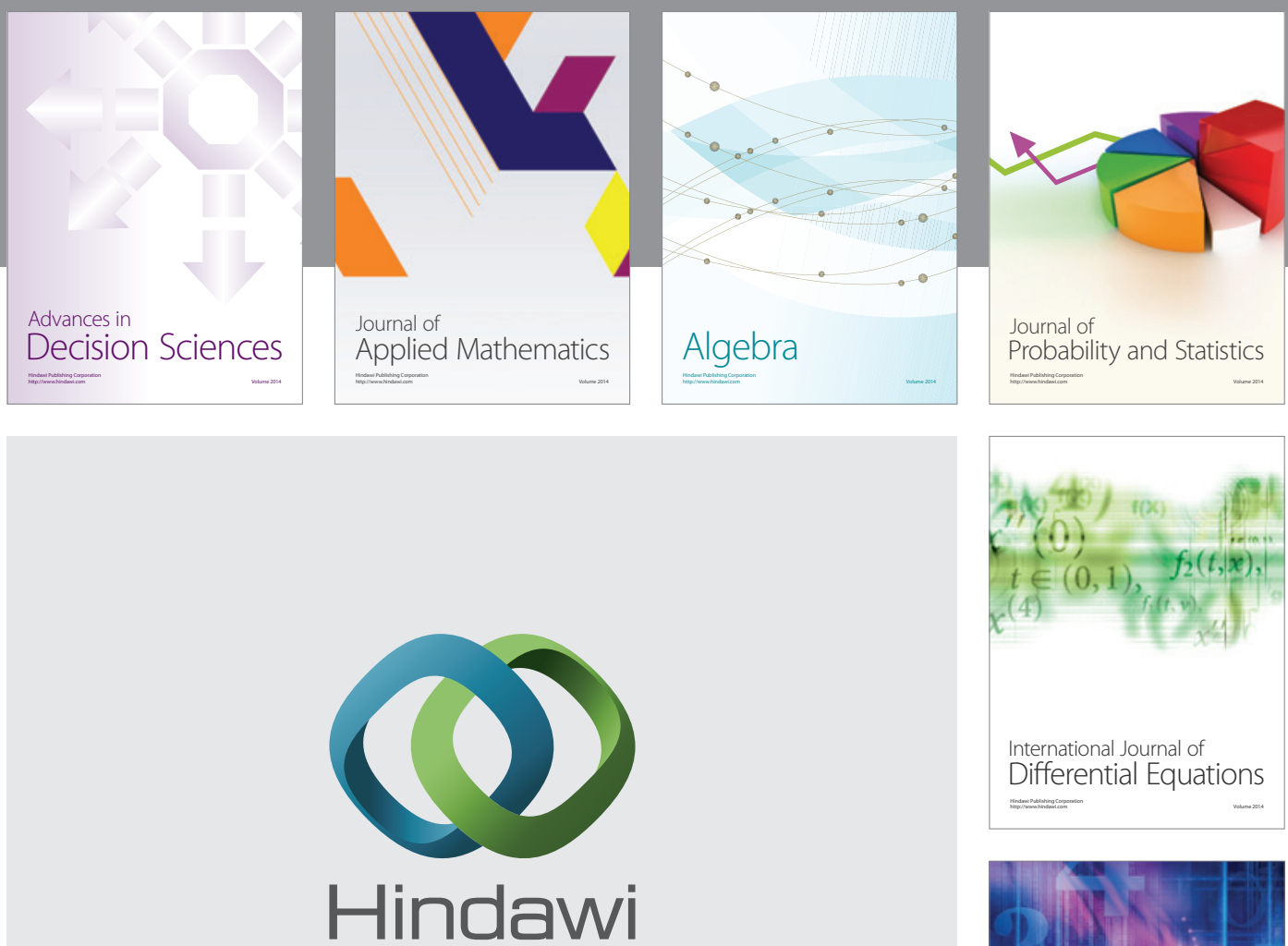

Submit your manuscripts at http://www.hindawi.com
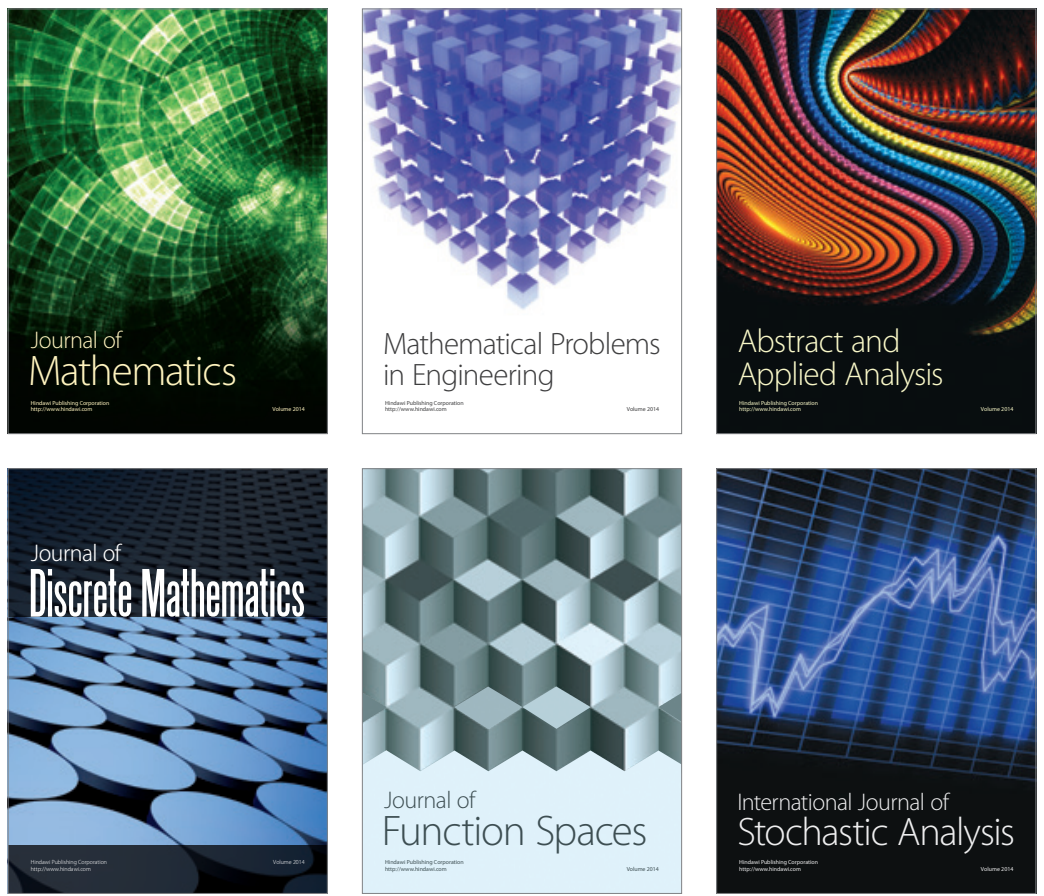

Journal of

Function Spaces

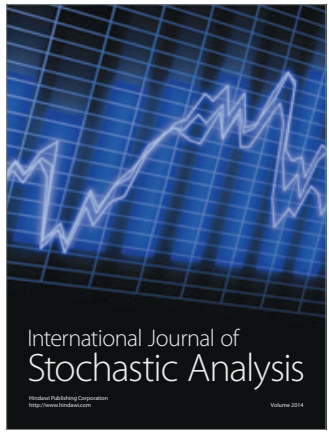

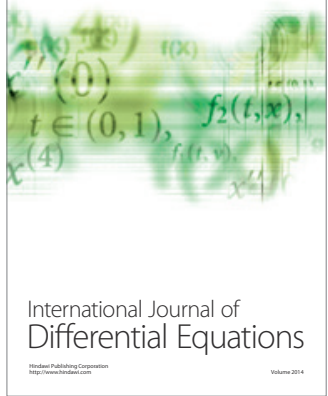
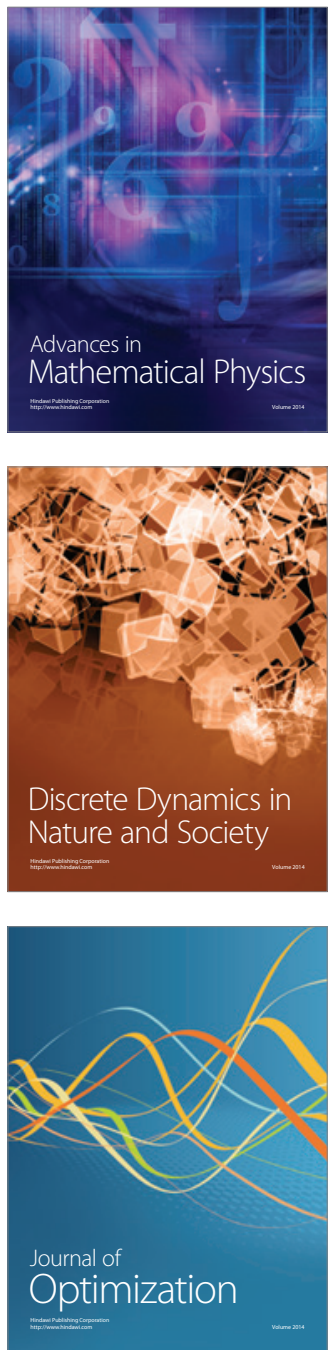